\title{
Audit of gastric residual volumes in an adult critical care setting
}

\author{
M. Currie, O. Mclaughlin, M. R. Ferguson, N. J. Flint, S. Gerty, C. Archdeacon and T. Lessells \\ University Hospitals of Leicester, Leicester LE15WW, UK
}

Many adult critical care units (ACCU) use a standard feeding protocol to commence enteral nutrition (EN). This often involves measuring gastric residual volumes (GRV); if the volume is $\geq 200 \mathrm{ml}$ the patient is considered to not be absorbing the feed ${ }^{(1)}$. Recently this protocol has been disputed as higher GRV can be present in the stomach than is measured and does not correlate well with increased aspiration risk $^{(2)}$.

A retrospective audit of GRV and delivery of EN was carried out for patients on the ACCU between October and December 2007. GRV were recorded for up to 14 days of ACCU stay. Other data collected included volume of feed delivered, volume prescribed, the use of prokinetics (metoclopramide \pm erythromycin) and other symptoms consistent with intolerance of EN (constipation, vomiting and high abdominal pressures/distension).

During this period, 214 patients were admitted to the ACCU; of these, 53 patients met the inclusion criteria (excluded if on ACCU for $<48 \mathrm{~h}$, or fed via a percutaneous tube, Parenteral Nutrition (PN) or case notes unavailable). Of these 53 patients, 28 (13 surgical and 15 medical) were fed via Nasogastric tube; the remaining patients were either eating and drinking or received no nutrition during this time.

The EN was turned off in $67 \%$ of patients (all surgical patients) between 2 and 8 times. The reason in $68 \%$ of cases was due to feed intolerance, $43 \%$ of patients received less than $50 \%$ of feed volume prescribed and $11 \%$ received less than $10 \%$ of feed prescribed. Prokinetics were used in $49 \% ; 21 \%$ being on dual therapy. Abdominal distension was present in nine patients (32\%) and GRV in this group varied: $55 \%$ had total GRV of less than $1200 \mathrm{ml}$ in $24 \mathrm{~h}$. Thirty-two percent of patients showed an unusual pattern of GRV with minimal or nil aspirates, followed by aspirates of greater than $200 \mathrm{ml}$ or vomiting. One patient aspirated while feeding during this period and the GRV of this patient was less than $600 \mathrm{ml}$ daily; 3 patients $(11 \%)$ went on to PN.

To conclude, using a $200 \mathrm{ml} \mathrm{GRV}$ as an indicator of feed tolerance appears to hinder the administration of EN. We believe that from our small audit a standard feeding protocol using $400 \mathrm{ml}$ cut-off point for GRV as a marker of tolerance would lead to a greater delivery of EN. Forty-seven percent of patients would have benefited from this higher GRV cut-off point ( 2 patients potentially avoiding PN) and in the remaining $53 \%$ patients this would have made no difference but would not have been detrimental.

1. Raper S \& Maynard N (1992) Br J Nurs 1, 273-280.

2. McClave SA, Lukan JK, Stefater JA et al. (2005) Crit Care Med 33, 324-330. 